\title{
Flexible and unique representations of two-digit decimals
}

\author{
Li Zhang ${ }^{1,2}$, Min Chen ${ }^{1,2}$, Chongde Lin ${ }^{3}$, Denes Szücs ${ }^{4}$
}

${ }^{1}$ Faculty of Psychology, Southwest University, China

${ }^{2}$ Key Laboratory of Cognition and Personality, Ministry of Education, China

${ }^{3}$ Institute of Developmental Psychology, Beijing Normal University, China

${ }^{4}$ Department of Experimental Psychology, Centre for Neuroscience in Education, University of Cambridge, UK

\section{Acknowledgments}

This study was supported by the Open Research Fund of the State Key Laboratory of Cognitive Neuroscience and Learning (CNLYB1208). We thank Francesca Hill for proofreading. We thank the reviewers for their helpful and constructive comments. Correspondence concerning this article should be addressed to Li Zhang, lilyking_0717@126.com, or Chongde Lin, linchongde@263.net 


\begin{abstract}
We examined the representation of two-digit decimals through studying distance and compatibility effects in magnitude comparison tasks in four experiments. Using number pairs with different leftmost digits, we found both the second digit distance effect and compatibility effect with two-digit integers but only the second digit distance effect with two-digit pure decimals. This suggests that both integers and pure decimals are processed in a compositional manner. In contrast, neither the second digit distance effect nor the compatibility effect was observed in two-digit mixed decimals, thereby showing no evidence for compositional processing of two-digit mixed decimals. However, when the relevance of the rightmost digit processing was increased by adding some decimals pairs with the same leftmost digits, both pure and mixed decimals produced the compatibility effect. Overall, results suggest that the processing of decimals is flexible and depends on the relevance of unique digit positions. This processing mode is different from integer analysis in that two-digit mixed decimals demonstrate parallel compositional processing only when the rightmost digit is relevant. Findings suggest that people probably do not represent decimals by simply ignoring the decimal point and converting them to natural numbers.
\end{abstract}

Keywords: Two-digit decimals; Parallel and sequential processing Distance effect; Numerical cognition; Magnitude representation 


\section{Introduction}

In everyday life we mostly encounter multi-digit numbers (Nuerk \& Willmes, 2005). Hence, it is important to understand how multi- digit numbers are represented and processed in the human mind (Meyerhoff, Moeller, Debus, \& Nuerk, 2012). However, our understanding of the mental representation of two-digit numbers remains incomplete (see Nuerk \& Willmes, 2005 for review). So far, most studies have explored the processing of two-digit integers by observing the distance effect and the compatibility effect. The distance effect refers to the fact that speed and accuracy increase with numerical distance between the to-be-compared numbers (Hinrichs, Yurko, \& Hu, 1981; Moyer \& Landauer, 1967). The compatible effect refers to the fact that unit-decade compatible number pairs are responded to faster and more accurately than unit-decade incompatible pairs (e.g., for 52-47, 5 N 4 but 2 b 7) (Nuerk, Weger, \& Willmes, 2001). To date, only two studies have addressed the processing of two-digit decimals. Here we examined whether findings relating to the processing of integers can be extended to two-digit decimals.

According to the holistic model of processing two-digit integers, people represent two-digit integers on a mental number line as an integrated entity (e.g., Dehaene, Dupoux, \& Mehler, 1990; Hinrichs et al., 1981; Reynvoet \& Brysbaert, 1999). For example, the seminal paper by Dehaene et al. (1990) compared two-digit numbers with a fixed standard. Even when the unit and decade digits of each target were presented asynchronously, reaction times decreased with the holistic target-standard distance; this is compatible with the holistic model.

Challenging the holistic model, the compositional model posits that the decade and unit digits of two-digit numbers are represented separately (compositional representation). This model is based on the place-value structure of Arabic numbers with the value of digits being determined by their position within the digit string (e.g., McCloskey, 1992; Szücs \& Soltész, 2010; Verguts \& De Moor, 2005). The so-called unit-decade compatibility effect provides robust evidence for this model (Nuerk et al., 2001). Nuerk et al. (2001) asked participants to choose the larger of a pair of two-digit 
numbers. Participants responded more quickly for pairs that were unit-decade compatible than for those that were unit-decade incompatible. This suggests separate processing of unit and decade digits and is not in line with the purely holistic model. Hence, it was concluded that not only the whole magnitude but also the magnitude of the unit and decade digits were represented (Nuerk \& Willmes, 2005; Nuerk et al., 2001).

So far, the compatibility effect has been observed in several studies (e.g., Ganor-Stern \& Tzelgov, 2011; Gazzellini \& Laudanna, 2011; Moeller, Fischer, Nuerk, \& Willmes, 2009; Nuerk, Moeller, Klein, Willmes, \& Fischer, 2011; Nuerk \& Willmes, 2005). Yet, the mode of compositional processing of two-digit numbers can be either parallel or sequential (e.g., Meyerhoff et al., 2012; Zhang \& Wang, 1995). Parallel processing assumes that each digit is represented separately and similarly. Each digit, even if irrelevant to the task influences the numerical comparison performance. Most previous studies on two-digit numbers have revealed evidence to suggest that decades and units are processed in parallel (Meyerhoff et al., 2012; Moeller, Fischer, et al., 2009; Moeller, Nuerk, \& Willmes, 2009; Nuerk \& Willmes, 2005; Nuerk et al., 2001; Verguts \& De Moor, 2005; Zhang \& Wang, 2005). In these studies, the compositional processing in parallel is generally indicated by a positive compatibility effect. That is, compatible trials are processed faster than incompatible ones (Ganor-Stern, Pinhas, \& Tzelgov, 2009; Meyerhoff et al., 2012; Nuerk, Kaufmann, Zoppoth, \& Willmes, 2004). In contrast, sequential processing refers to the fact that two-digit Arabic numerals are compared digit by digit sequentially from left to right. It is believed that the leftmost digits are more important and therefore processed be- fore those appearing on the right. Evidence for sequential processing mostly comes from studies on four- or six-digit integers (Meyerhoff et al., 2012; Poltrock \& Schwartz, 1984). For example, Meyerhoff et al. (2012) found that four-and six-digit numbers were divided into digit chunks consisting of 2 to 4 digits; chunks were processed sequentially while digits were processed in parallel within chunks. Similar to several studies (Ganor-Stern et al., 2009; Nuerk, Kaufmann, et al., 2004), Meyerhoff et al. (2012) found evidence for sequential processing through a negative compatibility effect, that is, incompatible trials were processed faster than compatible trials. In addition, Szücs and Soltész (2010) 
suggested that four-digit complex numbers were processed in parallel rather than sequentially.

Recent work indicates that the compatibility effect is influenced by the relevance of the rightmost unit digit processing during the comparison task (Huber, Mann, Nuerk, \& Moeller, 2013; Macizo \& Herrera, 2011). In their study, Macizo and Herrera (2011) heightened the relevance of unit processing by increasing the percentage (i.e., 20\%, 50\%, and 70\%) of same-decade filler items (e.g., 52_58) in the stimulus set. Their results showed that when the unit digits became more relevant than the decade digits (70\% same-decade comparisons) the compatibility effect shifted from negative to positive. A recent study by Huber et al. (2013) provided the first eye-tracking evidence to indicate that the compatibility effect increases with the percentage of same-decade filler items (i.e., 25\%, 50\%, and 75\%) which was accompanied by less fixations on tens and more fixations on units.

Taken together, it is still debatable whether compositional representation is involved in the processing of two-digit numbers and whether such processing is parallel or sequential. While these issues have been addressed extensively with integers, fewer studies have examined the processing of two-digit decimals in adults. Decimals refer to a decimal expression of rational numbers, which are denoted by the decimal point (“.”) and sequences of digits (e.g., 0.32 and 3.2). Although “32”, “0.32”, and “3.2” are visually very similar, they indicate entirely different numerical magnitudes. To our knowledge, only two previous studies have investigated the processing of decimals (Cohen, 2010; Varma \& Karl, 2013). Cohen (2010) compared the processing of two-digit integers and decimals between 0 and 1 in numerical comparison tasks. Data revealed that the numerical distance is the primary variable controlling participants' RTs for the comparison of integers. However, the physical similarity between the tenths place of the standard and the probe was found to be the primary variable controlling participants' RTs for the comparison of decimals. It was concluded that the processing of decimals is different from that of integers. Varma and Karl (2013) revealed that decimals between 0 and 1 produced the tenths-hundredths compatibility effect and their tenth and hundredth components are processed in parallel when comparing two 
decimals.

Given the small number of relevant studies, the present study aimed to provide new literature to clarify the nature of mental representation of two-digit decimals. Although two studies have addressed the processing of two-digit decimals between 0 and 1 , here we extend this previous research by exploring the processing of two-digit decimals larger than 1 as well as decimals between 0 and 1 . Two-digit decimals between 0 and 1 can be called pure decimals which have two decimal places with 0 in the integer place (e.g., 0.54). Two-digit decimals larger than 1 can be called mixed decimals which consist of one integer place plus one decimal place (e.g., 5.4). Clarifying how mixed decimals are processed by the human brain is theoretically important because findings can provide insight into whether the understanding of integers can be extended to all kinds of decimals. In addition, this study aimed to examine whether the findings of Macizo and Herrera (2011) and Huber et al. (2013) can be generalized to the comparison of decimals. For the first time, we empirically tested whether there was an increasing compatibility effect when the relevance of the rightmost digit processing in the decimal comparison increased.

In our study, if the hundredths or tenths distance (pure or mixed decimals) have significant influence on the comparison of two-digit decimals and a compatibility effect similar to the unit-decade compatibility effect in studies on integers can be observed, this may indicate that decimals are processed compositionally. Decade digits and whole numbers have a very high shared variance of numerical magnitude $(r=.97)$, thus the critical results should involve the unit digits (Zhou, Chen, Chen, \& Dong, 2008). Accordingly, we used the digit distance on the rightmost value position and the compatibility effect as the two main indicators of compositional processing. While both these indicators reflect that the rightmost digit is separately represented, the compatibility effect does this in an indirect way by revealing that the processing of the two digits interferes with each other. Furthermore, if compositional representation exists, parallel processing is indicated by a positive compatibility effect while sequential processing is associated with a negative compatibility effect (Ganor-Stern et al., 2009; Meyerhoff et al., 2012; Nuerk, Kaufmann, et al., 2004). 
To sum up, this study aimed to explore whether the processing of decimals was compositional and whether compositional processing, if it was found to exist, was parallel or sequential. In addition, the influence of the relevance of rightmost digit processing on the compatibility effect of pure and mixed decimals was investigated for the first time. Four experiments were conducted. Experiment 1 examined the processing of two-digit integers in Chinese subjects, in which we aimed to replicate the study by Nuerk et al. (2001) and to provide a comparison referent for decimals. Experiment 2 and 3 examined the processing of two-digit pure decimals and mixed decimals when there was no relevance of the rightmost digit processing in the comparison tasks, respectively. In contrast, Experiment 4 examined the processing of two-digit pure decimals and mixed decimals when there was relevance of the rightmost digit processing in the comparison tasks.

\section{Experiment 1}

In Experiment 1, a magnitude comparison task was used to address the specific processing of integers. Participants were asked to judge the larger of the two simultaneously presented integers.

\subsection{Methods}

\subsubsection{Participants}

Twenty-seven undergraduates were recruited from Southwest University, China. Their ages ranged from 18 to 26 years (mean age $=21.1$ years, $S D=1.7$ ). All subjects were of right handedness and had normal or corrected to normal eyesight. They gave written informed consent before the experiment. After the experiment, each participant was paid RMB 10 yuan.

\subsubsection{Materials and design}

The item set of Nuerk et al. (2001) was used and there were two hundred and forty two-digit integer pairs between 21 and 98. Single- digit numbers and two-digit numbers which had the same number in two digit places were ruled out. For each pair of numbers, the number differed in the first digit so that participants could make a decision by the first digit. The overall distance, each digit distance, problem size, and 
respective number word length of the stimulus groups across different conditions were matched both absolutely and logarithmically. Additionally, we assured that four different digits were presented and that the two numbers could not be divided by each other (see Table 1, Nuerk et al., 2001). In order to control for a possible order effect, each pair appeared once with the numerically larger number on the left side and once with the numerically smaller number on the left side.

A $2 \times 2 \times 2$ within participants design was used. The manipulated variables were decade distance (small: 1 to 3; large: 4 to 7 ), unit distance (small: 1 to 3; large: 4 to 8), and unit-decade compatibility (compatible vs. incompatible).

\subsubsection{Procedure}

Participants were asked to choose the larger of the paired two-digit integers which were simultaneously presented beside each other. They needed to press the "F" key if the left number was larger than the right number and press the "J" key if the right number was larger than the left number. All the stimuli were randomly presented.

Participants completed the magnitude comparison task individually in a sound attenuated small room, facing approximately $60 \mathrm{~cm}$ from the computer screen. All the stimuli were presented visually in black (Times New Roman, 28 point size) against a white background at the center of the 19 inch color monitor with a $75 \mathrm{~Hz}$ refresh rate. The resolution of the monitor was $800 \times 600$ pixels.

For each trial, a fixation point was first presented for $500 \mathrm{~ms}$. Then two numbers were presented $2.5 \mathrm{~cm}$ apart from the screen center until response or until $3000 \mathrm{~ms}$ had elapsed. After an interval of 500 ms, the next trial would begin. Before the formal experiment, there were 8 training trials. The formal experiment consisted of 480 trials with each block consisting of 80 trials. After each block, the subjects were allowed to take a break, which they could end at their own pace.

\subsubsection{Data analysis}

Reaction times from correctly responded trials were analyzed. For each participant, scores more than three standard deviations from the mean were excluded as outliers. Then the average RTs for each participant in each condition (a total of 8 conditions) were calculated. The data were subjected to a repeated-measures ANOVA 
with decade distance (small vs. large), unit distance (small vs. large), and unit- decade compatibility (compatible vs. incompatible) as within subjects factors.

\subsection{Results}

Table 1 shows the RTs and accuracy in each condition. The pattern of results in RTs analyses was identical to that produced in accuracy analyses, indicating that the decade distance, unit distance and compatibility had significant influence on the comparison of two-digit integers. The decade distance main effect was significant (RTs: $F(1,26)=$ 193.945, $p<.001, \mathrm{y}^{2}=.882$; Accuracy: $\left.F(1,26)=98.226, p<.001, \mathrm{y}^{2}=.791\right)$. Pairs with large decade distance were responded to $64 \mathrm{~ms}$ faster and $4.75 \%$ more accurately than pairs with small decade distance. The unit distance main effect was also significant $\left(\mathrm{RTs}: F(1,26)=9.472, p<.001, \mathrm{y}^{2}=.267\right.$; Accuracy: $F(1,26)=$ 5.836, $\left.p=.023, \mathrm{y}^{2}=.183\right)$. However, it was a reverse distance effect since pairs with small unit distance were responded to $8 \mathrm{~ms}$ faster and $0.62 \%$ more accurately than pairs with large unit distance. Finally, the compatibility main effect was significant $\left(\right.$ RTs: $F(1,26)=6.555, p=.017, \mathrm{y}^{2}=.201$; Accuracy: $F(1,26)=4.936, p$ $\left.=.035, \mathrm{y}^{2}=.160\right)$. Compatible number pairs were responded to $5 \mathrm{~ms}$ faster and $0.75 \%$ more accurately than incompatible pairs. No interaction reached significance.

\section{Table 1 about here}

\subsection{Discussion}

Experiment 1 demonstrated significant unit distance and compatibility effects. This provides evidence for a compositional representation of two-digit integers. Furthermore, the positive unit-decade compatibility effect in our study revealed that participants represented decade and unit digits in parallel. These results are consistent with previous studies (Meyerhoff et al., 2012; Moeller, Fischer, et al., 2009; Moeller, Nuerk, et al., 2009; Nuerk \& Willmes, 2005; Nuerk et al., 2001; Verguts \& De Moor, 2005).

Interestingly, we found a classical distance effect for decade digits but a reverse distance effect for unit digits. In fact, a similar finding was detected by Nuerk et al. (2001); a reverse distance effect was observed for unit digits, especially in the 
incompatible condition with participants performing $30 \mathrm{~ms}$ faster in the close distance than the far distance (see the Table 2, Nuerk et al., 2001). However, the reverse distance effect for unit digits was not discussed in their study. A further discussion of a classical distance effect for decade digits and a reverse distance effect for unit digits is presented in the General discussion.

\section{Experiment 2}

Experiment 1 showed that the comparison of two-digit integers involved compositional parallel processing. Integers and pure decimals are visually very similar. It has been found that when understanding a decimal (e.g., 0.32), both the correct referent (e.g., 0.32) and the incorrect natural number referent (e.g., 32) corresponding to the visually similar natural number expression are accessed in parallel (Varma \& Karl, 2013). Therefore, it is unclear whether the findings in Experiment 1 can be extended to two-digit pure decimals. To that end, this experiment was identical to Experiment 1 with one exception: the decimal point was moved forward two digits. We aimed to explore the processing of two-digit pure decimals by analyzing the distance effect and hundredths-tenths compatibility effect.

\subsection{Methods}

\subsubsection{Participants}

Twenty-one undergraduates (mean age $=22.6$ years, $\mathrm{SD}=1.7$ ) were recruited from Southwest University, China. Their ages ranged from 20 to 26 years. All of them were of right handedness. Other characteristics for these participants matched those in Experiment 1. All subjects had not participated in Experiment 1.

\subsubsection{Materials and design}

The materials were identical to Experiment 1 except that the deci- mal point was moved forward two digits. Thus, the number pairs for pure decimals were between 0.21 and 0.98. Likewise, a $2 \times 2 \times 2$ within participants design was used. The procedure was identical to that in Experiment 1. 


\subsection{Results}

Table 2 shows the RTs and accuracy in each condition. In RTs analyses, the tenths distance main effect was significant, $F(1,20)=450.203, p<.001, \mathrm{y}^{2}=.957$. Pairs with large tenths distance were responded to $66 \mathrm{~ms}$ faster than pairs with small tenths distance. The hundredths distance main effect was also significant, $F(1,20)=$ 11.711, $p=.003, \mathrm{y}^{2}=.369$. Similarly, a reverse pattern was observed: pairs with small hundredths distance were responded to $8 \mathrm{~ms}$ faster than pairs with large hundredths distance.

In accuracy analyses, the tenths distance effect was significant, $F(1,20)=$ $50.014, p=.001, \mathrm{y}^{2}=.714$. Pairs with large tenths distance were responded to $5.37 \%$ more accurately than pairs with small distance. In addition, a significant interaction of the tenths distance $\times$ the hundredths distance $\times$ compatibility was observed, $F(1,20)=$ 7.119, $p=.015, \mathrm{y}^{2}=.263$. Follow up analyses indicated that the tenths distance $\times$ the hundredths distance interaction was significant for incompatible pairs, $F(1,20)$ $=6.550, p=.019, \mathrm{y}^{2}=.247$, but not for compatible pairs $(p=.961)$. Post hoc t-tests showed that the hundredths distance effect was significant with the large tenths distance, $t(20)=2.364, p=.028$, but not with the small tenths distance $(p=.089)$.

In order to explain why no compatibility effect was observed in the above analyses, it might have been the case that one half showed a negative compatibility effect and the other half a positive compatibility effect resulting in a null effect overall (Meyerhoff et al., 2012). There- fore, we inspected the individual pattern of the compatibility effect. We conducted a one-way ANOVA with the compatibility effect as the independent variable and RTs as the dependent variable for each participant. The results revealed that only one participant showed a significant positive compatibility effect ( $p=.011$ ) and the other 20 participants showed neither a positive nor negative compatibility effect ( $p s>$.064). Therefore, the non-significant compatibility effect was not due to the offset between a positive and negative compatibility effect.

\subsection{Discussion}

This experiment showed that two-digit pure decimals were processed 
compositionally as revealed by the significant hundredths distance effect in RTs analyses. In addition, the significant interaction of the tenths distance, the hundredths distance and compatibility in accuracy analyses revealed the influence of hundredths digits on the comparison of pure decimals. The hundredths distance effect appeared only in incompatible pairs with large tenths distance. We suggest that when the tenths distance is large, participants will have more resources for hundredths digit processing because number pairs with large tenths distance are more easy to discriminate than pairs with small tenths distance. Meanwhile, in the tenths-hundredths-incompatible pairs, the two comparisons for the tenths and the hundredths lead to different and conflicting results (e.g., 52-47, $5>4$ but $2<7$ ). The conflicting results, subsequently, may make participants less confident in relying only on the number comparison in the tenths place. Consequently, in order to be represented, the digit in the hundredths place may be devoted to more resources compared to that in compatible number pairs.

In summary, two-digit pure decimals were processed composition- ally. This finding is similar to that for integers in Experiment 1. However, there are differences between pure decimals and integers. For integers, there was obvious evidence for parallel processing. In contrast, it is difficult to judge whether the compositional processing of pure decimals was parallel or sequential, since there was neither a positive compatibility effect nor a negative compatibility effect.

\section{Experiment3}

Experiment 2 suggests that compositional processing is involved in the comparison of two-digit pure decimals. As compared to integers, pure and mixed decimals are visually more similar. Experiment 3 examined whether the above findings can be generalized to two-digit mixed decimals. This is the first instance in which mixed decimals have been used as stimuli in a numeral comparison task.

\subsection{Methods}

\subsubsection{Participants}

Twenty-two undergraduates were recruited from Southwest University, China. Their ages ranged from 19 to 25 years (mean age $=21.7$ years, $S D=1.4$ ). All of them 
were of right handedness. Other characteristics for these participants were similar to those in Experiment 1. All subjects had not participated in Experiments 1 and 2.

\subsubsection{Materials}

The materials were the same as those in Experiment 1 except that the decimal point was moved forward one digit (also the same as those in Experiment 2 except that the decimal point was moved back- ward one digit). Therefore, mixed decimals number pairs range from 2.1 to 9.8 were compared. The procedure was identical to that in Experiment 1.

\subsection{Results}

Table 3 shows the RTs and accuracy in each condition. Only the unit distance had a significant effect on the comparison of mixed decimals. The unit distance main effect was significant $\left(\mathrm{RTs}: F(1,21)=264.789, p=.001, \mathrm{y}^{2}=.929\right.$; Accuracy: $F(1,21)$ $\left.=42.091, p=.001, \mathrm{y}^{2}=.667\right)$. Pairs with large unit distance were responded to 58 ms faster and 3.73\% more accurately than pairs with small unit distance. No significant tenths distance effect, compatibility effect and interaction were observed.

Similarly, we inspected the individual pattern of the compatibility effect. The RTs analyses revealed that only one participant showed a significant positive compatibility effect ( $p=.004$ ) and only one participant showed a significant negative compatibility effect $(p=.003)$. The remaining 20 participants showed neither a positive nor negative compatibility effect ( $p s>$.066). Therefore, the non-significant compatibility effect should not be due to the offset between positive and negative compatibility effects.

\section{Table 3 about here}

\subsection{Discussion}

This experiment did not show any evidence for the compositional representation of two-digit mixed decimals. The tenths distance effect was not significant in both RTs and accuracy analyses, suggesting that tenths digits in mixed decimals are not represented separately.

\section{Experiment 4}


In Experiments 2 and 3, neither pure nor mixed decimals produced the compatibility effect. This finding may be associated with the fact that all the to-be-compared number pairs were different in the leftmost digits (e.g., 0.28_0.57 and 2.8_5.7). As a result, participants were able to correctly solve the tasks by only comparing the leftmost digits and ignoring the rightmost digits. In other words, no observation of the compatibility effect may be due to the irrelevance of the rightmost digit processing during the comparison tasks.

In order to test the possible influence of the relevance of the right-most digit processing on the compatibility effect of pure and mixed decimals, this experiment increased the relevance of the rightmost digit processing by designing two filler item ratios (20\% and $70 \%)$. The two filler item ratios were chosen based on the finding that the obvious differences mainly existed between $20 \%$ and $70 \%$ ratio conditions (Macizo \& Herrera, 2011). For pure decimals, the filler trials had the same tenths digits (e.g., 0.52_0.58); for mixed decimals, the filler trials had the same hundredths digits (e.g., 5.2_5.8).

\subsection{Methods}

\subsubsection{Participants}

A total of 79 undergraduates were recruited from Southwest University, China. Their ages ranged from 18 to 25 years (mean age $=20.6$ years, $S D=1.5$ ). There were 3 left-handed and 76 right-handed participants. All the participants had normal or corrected to normal eyesight. They were randomly assigned to one of four groups with one group consisting of 19 participants and each of the other three groups of 20 participants. No significant age differences between the four groups were found 20 ( $p$ $=.549$ ). All the participants gave written informed consent before the experiment. After the experiment, each participant was paid RMB 10 yuan.

\subsubsection{Materials and design}

The experimental trials included 240 pairs between 0.21 and 0.98 used in Experiment 2, and 240 pairs between 2.1 and 9.8 used in Experiment 3. All these pairs were different in the leftmost digits. The filler trials had the same leftmost digits between 0.21 and 0.89 for pure decimals and between 2.1 and 8.9 for mixed decimals. 
For both pure and mixed decimals, there were two types of filler trial ratios: $20 \%$ and $70 \%$ corresponding to 60 and 560 filler trials respectively.

Thus, two between-participant variables included types of decimals (pure or mixed) and filler item ratio (20\% or $70 \%)$. Therefore, four groups of participants were created and each group was assigned to one task. The stimuli list of each task was divided into two blocks with the same number of experimental and filler trials in each block (120 experimental trials and 30 filler trials for 20\% ratio; 120 experimental trials and 280 filler trials for $70 \%$ ratio). The block order was counter- balanced across lists.

\subsubsection{Procedure}

The procedure was identical to Experiment 1 except that there were 20 training trials for each task. After one block, participants were allowed to take a break, which they could end at their own pace.

\subsection{Results}

In Experiment 4, only the 240 experimental trials were analyzed. To provide a fully comprehensive test of the influence of the relevance of the rightmost digit on the processing of decimals, we incorporated Experiments 2, 3, and 4 into a joint analysis2 with first digit distance, second digit distance, and compatibility as within-participant variables as well as filler item ratio $(0 \%, 20 \%, 70 \%)$ and types of decimals as between-participant variables. With no relevance of the rightmost digit processing, Experiments 2 and 3 actually involved $0 \%$ filler item ratio for pure and mixed decimals. Tables 4 and 5 show the RTs and accuracy in each condition in Experiment 4, separately.

In RTs analyses, there were significant main effects of the first digit distance, $F$ (1, $116)=11.452, p<.001, \mathrm{y}^{2}=.926$, of compatibility, $F(1,116)=139.858, p$ $<.001, \mathrm{y}^{2}=.547$, of filler item ratio, $F(1,116)=21.449, p<.001, \mathrm{y}^{2}=.270$, and of types of decimals, $F(1,116)=14.253, p<.002, \mathrm{y}^{2}=.109$. Importantly, there were significant interactions between the first digit distance $\times$ compatibility $\times$ filler item ratio, $F(1,116)=8.854, p<.001, \mathrm{y}^{2}=.132$, between the second digit distance $\times$ compatibility $\times$ filler item ratio, $F(1,116)=5.160, p=.007, \eta^{2}=.082$, and between the first digit distance $\times$ the second digit distance $\times$ filler item ratio, $F$ 
$(1,116)=7.046, p=.001, \mathrm{y}^{2}=.108$

Follow-up analyses showed that the three-way interaction between the first digit distance $\times$ compatibility $\times$ filler item ratio was due to the fact that the two-way interaction between the first digit distance $\times$ compatibility was significant for $70 \%$ filler item ratio, $F(1,39)=21.906, p<.001, y^{2}=.360$, but not for $20 \%$ and $0 \%$ filler item ratios ( $p s>.400)$. Post hoc $t$-tests indicated that the positive compatibility effect was significant in both small and large first digit distances, $t$ (39) $=10.945, t$ (39) $=9.240$, $p s<.001$, but it was larger for the small than for the large first digit distance. It should be noted that for $20 \%$ filler item ratio the compatibility main effect was also significant, $F(1,38)=25.344, p<.001, \mathrm{y}^{2}=.400$. In contrast, for $0 \%$ filler item ratio, the compatibility main effect was not significant $(p=.593)$.

The three-way interaction between the second digit distance $\times$ compatibility $\times$ filler item ratio was due to the fact that the two-way interaction between the second digit distance $\times$ compatibility was significant for $20 \%$ filler item ratio, $F(1,38)=$ $11.995, p=.001, \mathrm{y}^{2}=.240$, but not for $70 \%$ and $0 \%$ filler item ratios ( $p s>.113$ ). Post hoc $t$-tests indicated that the second digit distance was significant for compatible pairs, $t(38)=3.908, p$ b .001, but not for incompatible pairs $(p=.508)$. In contrast, for $0 \%$ filler item ratio, the second digit distance main effect was significant, $F(1,42)=$ 10.079, $p=.003, \mathrm{y}^{2}=.194$, irrespective of compatible or incompatible pairs.

The three-way interaction between the first digit distance $\times$ the second digit distance $\times$ filler item ratio was due to the fact that the two-way interaction between the second digit distance $\times$ the first digit distance was significant for $20 \%$ and $0 \%$ filler item ratios, $F(1,38)=11.860, p=.001, \mathrm{y}^{2}=.293, F(1,42)=3.956, p$ $=.053, \mathrm{y}^{2}=.086$, but not for $70 \%$ filler item ratio $(p=.202)$. Post hoc $t$-tests indicated that for $20 \%$ filler item ratio, the second digit distance was significant with the large first digit distance, $t(38)=5.360, p<.001$, but not with the small first digit distance $(p=.293)$. For $0 \%$ filler item ratio, the reverse pattern appeared: the second digit distance was significant with the small first digit distance, $t(38)=-3.215, p$ $=.003$, but not with the large first digit distance $(p=.304)$. In contrast, for $70 \%$ filler item ratio, the second digit distance main effect was not significant $(p=.077)$. 
In accuracy analyses, there were significant main effects of the first digit distance, $F(1,116)=317.160, p<.001, y^{2}=.732$, of compatibility, $F(1,116)$ $=85.169, p<.001, \mathrm{y}^{2}=.423$, of filler item ratio, $F(1,116)=10.028, p=.001$, $\mathrm{y}^{2}=.147$. In addition, there was a significant interaction between the first digit distance $\times$ compatibility $\times$ filler item ratio, $F(1,116)=17.589, p<.001, \mathrm{y}^{2}=.233$, and between the first digit distance $\times$ the second digit distance $\times$ filler item ratio, $F$ $(1,116)=5.216, p=.007, \mathrm{y}^{2}=.083$.

Follow-up analyses showed that the three-way interaction between the first digit distance $\times$ compatibility $\times$ filler item ratio was due to the fact that the two-way interaction between the first digit distance $\times$ compatibility was significant for $20 \%$ and $70 \%$ filler item ratios, $F(1,38)=9.029, p=.005, \mathrm{y}^{2}=.192, F(1,38)=38.282$, $p<.001, \mathrm{y}^{2}=.495$, but not for $0 \%$ filler item ratio $(p=.567)$. Post hoc $t$-tests indicated that the compatibility effect was significant only with the small first digit distance for $20 \%$ filler item ratios, $t(38)=3.379, p=.002$. However, for $70 \%$ filler item ratio, the compatibility effect was significant with both small and large first digit distances, $t(39)=8.463, t(39)=5.845$, $p s<.001$, but it was larger with the small first digit distance than with the large first digit distance. In addition, for $0 \%$ filler item ratio, the compatibility main effect was not significant $(p=.982)$.

Similar to RTs results, the three-way interaction between the first digit distance $x$ the second digit distance $\times$ filler item ratio was due to the fact that the two-way interaction between the second digit distance $\times$ the first digit distance was significant for $20 \%$ and $0 \%$ filler item ratios, $F(1,38)=5.038, p=.031, \mathrm{y}^{2}=.117, F(1,42)$ $=4.757, p=.035, \mathrm{y}^{2}=.102$, but not for $70 \%$ filler item ratio $(p=.299)$. Post hoc $t$-tests indicated that for $20 \%$ filler item ratio, the second digit distance was significant with the small first digit distance, $t(38)=2.409, p=.021$, but not with the large first digit distance $(p=.917)$. For $0 \%$ filler item ratio, the second digit distance was significant with the large first digit distance, $t(42)=3.028, p=.004$, but not with the small first digit distance ( $p=.171$ ). In contrast, for $70 \%$ filler item ratio, the second digit distance main effect was not significant $(p=.724)$.

Taken together, it seems that the compatibility effect and the second digit distance 
effect were different among $0 \%, 20 \%$ and $70 \%$ filler item ratios. In order to confirm this finding, we computed the size of compatibility effect for each ratio. The size of compatibility effect on RTs or ac- curacy was computed by subtracting the RTs or accuracy on compatible trials from the RTs or accuracy on incompatible trials and then dividing by the RTs or accuracy on incompatible trials. On the other hand, we computed the size of distance effect for each ratio (Sasanguie, Defever, Van den Bussche, \& Reynvoet, 2011). The size of distance effect on RTs or accuracy was computed by subtracting the RTs or accuracy on large distance trials from the RTs or accuracy on small distance trials and then dividing by the RTs or accuracy on the small distance trials.

An ANOVA was then conducted with filler item ratio as an independent variable and the size of compatibility effect as the dependent variable. Results showed that the main effect of filler item ratio was significant $(\mathrm{RTs}: F(1,119)=54.599, p<.000$, $\mathrm{y}^{2}=.479$; Accuracy: $\left.F(1,119)=43.501, p<.001, \mathrm{y}^{2}=.422\right)$. The size of compatibility effect was biggest for the 70\% filler item ratio (RTs: 0.071; Accuracy: -0.073 ) and smallest for the $0 \%$ filler item ratio (RTs: 0.001; Accuracy: -0.0002 ). For the $20 \%$ filler ratio, the size of compatibility effect was in the middle (RTs: 0.028; Accuracy: -0.017).

An ANOVA with filler item ratio as an independent variable and the size of the second digit distance effect as the dependent variable was conducted. The RTs results showed that the main effect of filler item ratio was significant, $F(1,119)=8.162, p$ $<.000, \mathrm{y}^{2}=.121$. The size of the second digit effect for the $0 \%$ filler item ratio $(-0.0084)$ significantly differed from that for $20 \%$ and $70 \%$ filler item ratios $(0.0088$ and 0.0067). However, there was no significant difference between $20 \%$ and $70 \%$ filler item ratios. We noticed that the size of the second digit effect for the $0 \%$ filler item ratio (-0.0084) was negative. This finding is consistent with Experiment 2 and revealed a reverse second digit distance effect. Finally, the accuracy analysis did not show a significant main effect of the second digit distance. 


\subsection{Discussion}

The joint analyses showed that both pure and mixed decimals produced the positive compatibility effect when the relevance of the rightmost digit processing increased by adding 20\% and 70\% filler trials in the stimuli list. In particular, when the rightmost digit processing became more relevant than the leftmost digit processing with $70 \%$ filler trials, the positive compatibility effect was much stronger. The joint analyses also confirmed that with no relevance of the rightmost digit as in Experiments 2 and 3, no compatibility effect was observed. These results suggest that the absence of a compatibility effect in Experiments 2 and 3 may be closely associated with the irrelevance of the rightmost digit processing. Taken together, the processing of pure and mixed decimals is modulated by the relevance of the rightmost digit processing in the comparison tasks. When there was high relevance of the rightmost digit processing in the comparison tasks, the processing of pure and mixed decimals was compositional and in parallel. However, when there was no relevance of the rightmost digit processing in the comparison tasks, no strong evidence of parallel compositional processing could be observed in pure decimals as revealed in Experiment 2 and no strong evidence of compositional processing could be observed in mixed decimals as revealed in Experiment 3.

In addition, our analyses showed that the second digit distance effect was modulated by the relevance of the rightmost digit processing. How- ever, unlike the compatibility effect, the second digit distance effect was negatively affected by the relevance of the rightmost digit processing. The second digit distance effect was significant for the $0 \%$ filler item ratio, but not for the $70 \%$ filler item ratio. It seems that there is a dissociation between the compatibility effect and the second digit distance effect. The underlying reason may be that, when the compatibility effect increased, fewer resources were allocated to the representation of the second digit. The increased compatibility effect reflects the fact that the first digit is more strongly interfered by the second digit with the increased relevance of the rightmost digit. As a result, more resources are required for 
participants to inhibit the interference from the second digit in order to give correct answers in the comparison task. Thus, fewer resources are available for the representation of the second digit. In particular, the reliable and classic distance effect may involve more refined, algorithm-based process (Tzelgov, Meyer, \& Henik, 1992).

\section{General discussion}

We examined the processing of two-digit integers, two-digit pure decimals and two-digit mixed decimals in a magnitude comparison task without fixed standards. In line with previous studies (Moeller, Fischer, et al., 2009; Moeller, Nuerk, et al., 2009; Nuerk \& Willmes, 2005; Nuerk et al., 2001), results confirmed the compositional representation of two-digit integers. Most importantly, our study revealed that with no relevance of the rightmost digit processing in the comparison tasks, pure decimals were represented compositionally while we did not detect compositional representation for mixed decimals. However, with relevance of the rightmost digit processing in the comparison tasks, both two-digit pure and mixed decimals were processed compositionally and in parallel.

\subsection{The processing of two-digit pure decimals}

Experiments 1 and 2 revealed that with no relevance of the rightmost digit processing in the comparison tasks, the processing of two-digit pure decimals was similar to that of integers. For example, the classical distance effect was shown at the first digit and the reverse distance effect was shown at the second digit for both integers and pure decimals. We suggest that there are different processing mechanisms for the first and second digits of two-digit integers and pure decimals.

Previous studies have typically reported a reverse distance effect in order judgment tasks; such tasks usually require that participants judge whether two or three numbers are in the correct order (Franklin \& Jonides, 2009; Franklin, Jonides \& Smith, 2006, 2009; Turconi, Campbell, \& Seron, 2006). An fMRI study by Franklin and Jonides (2009) observed the typical distance effect in a magnitude comparison task but a reverse distance effect in an order-judgment task. The intraparietal sulcus (IPS) was activated in both the magnitude and order tasks but participants seemed to use different strategies 
for the two tasks. The authors suggested that a comparison mechanism was used in the magnitude comparison task but that a scanning mechanism was used in the order task leading to a reverse distance effect (Franklin \& Jonides, 2009; Franklin et al., 2009).

Accordingly, in our study, it is likely that the comparison of two-digit integers and pure decimals involved a comparison process for the first digit and a scanning process for the second digit. In magnitude comparison tasks, digits are hard to discriminate when their distance is small so that reactions times are longer for small distances than for large distances. In contrast, in order scanning processing, participants need to scan more numbers between the two presented numbers when the distance is large (e. g., scanning of numbers 2 to 4 require less time than scanning the range of 2 to 8 ) so that it takes more time for the large distance than for the small distance. However, it remains unknown as to why the scanning process is only present for the second digit. We suggest that participants might realize that they can make a decision based on the first digit only and it is unnecessary to compare the magnitude of the second digit. Therefore, they just scan the order information of the second digit. Indeed, the first digits of each comparison number pair in the present study were different, which meant that participants could make a decision without comparing the second digits.

In summary, when there was no relevance of the rightmost digit processing in the comparison tasks, the two digits of both two-digit integers and pure decimals were represented separately although possibly relying on different processing mechanisms. However, there are some differences between two-digit integers and pure decimals. With the positive compatibility effect as an indicator, this study showed strong evidence for the parallel processing of two-digit integers but not for two-digit pure decimals. This finding demonstrates that although the digits of pure decimals may be processed separately, the less relevant hundredths digits did not produce a Stroop-like interference to the more relevant tenths digits. In contrast, for integers, the less relevant unit digits came to produce a Stroop-like interference to the more relevant decade digits. Similarly, in the study by Varma and Karl (2013), although decimals produced a positive compatibility effect, the effect was less obvious than in integers. That is, decimals showed less interference than integers. The possible explanation is that the 
digits far from the decimal point were more related to precision rather than to magnitude. As one moves successively away from the decimal point, less information about quantity is provided, although greater precision is attained (Cohen, 2010). Therefore, the digits far from the decimal point were less important and involved less processing effort than those close to the decimal point in the magnitude comparison. Most notably, in Experiment 2 all comparisons could be made based only on the tenths place, therefore participants might have paid less attention to the hundredths place.

However, with relevance of the rightmost digit processing by mixing pure decimal pairs differing in their first digits with pure decimal pairs with the same first digits, Experiment 4 found strong evidence for the parallel compositional processing of pure decimals, as revealed by the large compatibility effect. These results are basically consistent with the finding from two-digit integers by Macizo and Herrera (2011). The strong positive compatibility effect in Experiment 4 as compared to no comparability effect in Experiment 2 may be explained by the requirement of more conflict control in Experiment 4. In Experiment 4, the processing of the rightmost hundredths became very relevant because hundredths were processed necessarily to solve the comparison task with the same leftmost digit pairs. Following the rationale of the study by Macizo and Herrera (2011), the increased relevance of the rightmost hundredths would make participants focus on the hundredths representation in the comparison task. Furthermore, the associative learning during the within-tenths comparison with the same tenths and different hundredths (e.g., 0.52_0.58) would reinforce the connections between the hundredths representation and the stimulus proper- ties in the task, which would produce conflict when between-tenths numbers (e.g., 0.52_0.38) were tenths-hundredths incompatible. Taken together, the results from Experiments 2 and 4 indicate that the processing of pure decimals is flexible and depends on the specific characteristics of the stimuli at hand.

\subsection{The processing of two-digit mixed decimals}

For the first time, our study provides information on how people represent and process mixed decimals. Unlike two-digit integers and pure decimals, there was little evidence for compositional representation when there was no relevance of the tenths 
processing in the comparison tasks. A possible explanation may be that the magnitude of the left digits closely approximated the whole magnitude of mixed decimals. Specifically, for a mixed decimal 5.4, the magnitude of the left digit is 5 and the difference between 5 and 5.4 is very small. For an integer 54, the magnitude of the left digit is also 5 but the difference between 5 and 54 is 49 . For a pure decimal 0.54 , the magnitude of the left digit is also 5 but the difference between 5 and 0.54 is 4.46 . When the difference between the magnitude of the left digits and the whole magnitude of two-digit numbers is larger, participants are probably more likely to use compositional processing to reinforce holistic processing. Indeed, decomposition of a two-digit number allows one to "recycle" the strong connections from single-digit numbers so that two-digit numerical comparison can be performed without error (Verguts \& De Moor, 2005).

For mixed decimals, the difference between the magnitude of the left digits and the whole magnitude is so small that it makes no difference whether participants rely on holistic processing or on compositional processing of digits on the left. Therefore, we suggest that participants may be confident in generating correct answers solely by holistic processing without resorting to compositional processing. For two-digit integers, the difference between the magnitude of the left digits and the whole magnitude is the largest when compared to decimals. In order to perform the numerical comparison without error, participants seem to combine holistic and compositional processing in order to achieve higher accuracy. It should be noted that such combined holistic and compositional processing may be restricted to small numbers with two digits and may be reasonably familiar to educated adults and/or to those who have adequate cognitive resources for such an activity. In addition, given that the holistic magnitude of mixed decimals correlates highly with unit magnitude ( $\mathrm{r}$ $=.97$ ), a possible explanation as to why no compatibility effect was found may lie in the fact that only the unit digit was processed separately. The "decimal point” of mixed decimals may be a direct visual source. To form a representation, participants would process all important and necessary information before the point or on the left of the point while all the information after the point or on the right of the point is not 
important and can be ignored.

Taken together, with no relevance of the rightmost digit processing, the processing of decimals is different from that of integers, regardless of pure or mixed decimals. In general, this conclusion is consistent with the studies of Cohen (2010) and Varma and Karl (2013), demonstrating the uniqueness of decimal representation. Our findings challenge the conversion hypothesis which purports that people convert decimals to natural number expressions by ignoring decimal points and compare the corresponding natural number referents.

Interestingly, similar to pure decimals, with relevance of the right- most digit processing in Experiment 4, the processing of mixed decimals was compositional and in parallel. Relating to this, it was likely that with increased relevance of the tenths processing, the strong connections between the tenths representation and the stimulus properties appear to produce conflict when between-unit numbers with different unit digits (e.g., 5.3_2.7) were unit-tenths incompatible. To sum up, the results from Experiments 3 and 4 indicate that the processing of mixed decimals is sensitive to the relevance of the rightmost digit processing in the comparison tasks. The representation of mixed decimals is also flexible and depends on the specific characteristics of the stimuli at hand. The flexible representation of both pure and mixed decimals is similar to the phenomenon that the same two numbers are responded faster when presented in a smaller number range than in a larger number range(Pinhas, Pothos, \& Tzelgov, 2013). Also, it is similar to the finding that the same number was located differently depending on the neighboring numbers when participants were asked to locate the larger or smaller number in a pair on a horizontal line representing the interval from 0 to 10 (Shaki \& Fischer, 2013). It seems that the representation of both simple and complex numbers is constructed online in intentional processing of numerical magnitude. Future studies should explore whether the automatic processing and representation of simple and complex numbers depends on the specific characteristics of the stimuli at hand.

Finally, it should be noted that there is one cultural difference in the expression of decimals. In China and in North America, the decimal symbol is the point (.) while in some of Europe and South America, the decimal symbol may be the comma (,). It is 
likely that the processing of decimals (especially mixed decimals) which employ a comma as a decimal mark will greatly differ from that of integers. This may be because a comma provides a more visually obvious means of separating the integer from the decimal. However, this hypothesis remains to be tested in future studies.

\section{Conclusion}

To summarize, we showed that with no relevance of the rightmost digit processing in the comparison tasks, both two-digit integers and pure decimals were processed compositionally, but two-digit pure decimals did not show strong evidence for parallel compositional processing as compared to two-digit integers. Moreover, for the first time, our study revealed that there was little evidence for the compositional representation of mixed decimals with no relevance of the rightmost digit processing. However, with relevance of the rightmost digit processing, both pure and mixed decimals were processed compositionally and in parallel.

\section{References}

Cohen, D. J. (2010). Evidence for direct retrieval of relative quantity information in a quan- tity judgment task: Decimals, integers, and the role of physical similarity. Journal of Experimental Psychology: Learning, Memory, and Cognition, 36(6), $1389-1398$.

Dehaene, S., Dupoux, E., \& Mehler, J. (1990). Is numerical comparison digital? Analogical and symbolic effects in two-digit number comparison. Journal of Experimental Psychology: Human Perception and Performance, 16(3), 626-641.

Franklin, M. S., \& Jonides, J. (2009). Order and magnitude share a common representation in parietal cortex. Journal of Cognitive Neuroscience, 21(11), 2114-2120.

Franklin, M. S., Jonides, J., \& Smith, E. E. (2006, November). Distance effects in a task with numbers and months. A poster presented at the meeting of the Psychonomics Society, Houston, TX.

Franklin, M. S., Jonides, J., \& Smith, E. E. (2009). Processing of order information for numbers and months. Memory \& Cognition, 37(5), 644-654. 
Ganor-Stern, D., Pinhas, M., \& Tzelgov, J. (2009). Comparing two-digit numbers: the importance of being presented together. The Quarterly Journal of Experimental Psychology, 62(3), 444-452.

Ganor-Stern, D., \& Tzelgov, J. (2011). Across-notation automatic processing of two-digit numbers. Experimental Psychology, 58(2), 147-153.

Gazzellini, S., \& Laudanna, A. (2011). Digit repetition effect in two-digit number comparison. Zeitschrift für Psychologie/Journal of Psychology, 219(1), 30-36.

Hinrichs, J. V., Yurko, D. S., \& Hu, J. M. (1981). Two-digit number comparison: use of place information. Journal of Experimental Psychology: Human Perception and Performance, 74(4), 890-901.

Huber, S., Mann, A., Nuerk, H. -C., \& Moeller, K. (2013). Cognitive control in number magnitude processing - evidence from eye-tracking. Psychological Research. http:// dx.doi.org/10.1007/s00426-013-0504-X.

Macizo, P., \& Herrera, A. (2011). Cognitive control in number processing: Evidence from the unit-decade compatibility effect. Acta Psychologica, 136(1), 112-118.

McCloskey, M. (1992). Cognitive mechanisms in numerical processing: Evidence from acquired dyscalculia. Cognition, 44(1), 107-157.

Meyerhoff, H. S., Moeller, K., Debus, K., \& Nuerk, H. -C. (2012). Multi-digit number processing beyond the two-digit number range: A combination of sequential and parallel processes. Acta Psychologica, 140(1), 81-90.

Moeller, K., Fischer, M. H., Nuerk, H. -C., \& Willmes, K. (2009). Sequential or parallel decomposed processing of two-digit numbers? Evidence from eye-tracking. The Quarterly Journal of Experimental Psychology, 62(2), 323-334.

Moeller, K., Nuerk, H. -C., \& Willmes, K. (2009). Internal numbers magnitude representa- tion is not holistic, either. European Journal of Cognitive Psychology, 21(5), 672-685.

Moyer, R. S., \& Landauer, T. K. (1967). Time required for judgments of numerical inequality. Nature, 215(5109), 1519-1520.

Nuerk, H. -C., Kaufmann, L., Zoppoth, S., \& Willmes, K. (2004). On the 
development of the mental number line. More or less or never holistic with increasing age. Developmental Psychology, 40(6), 1199-1211.

Nuerk, H. -C., Moeller, K., Klein, E., Willmes, K., \& Fischer, M. H. (2011). Extending the mental number line. Zeitschrift für Psychologie/Journal of Psychology, 219(1), 3-22.

Nuerk, H. -C., Weger, U., \& Willmes, K. (2001). Decade breaks in the mental number line? Putting the tens and units back in different bins. Cognition, 82(1), B25-B33.

Nuerk, H. -C., \& Willmes, K. (2005). On the magnitude representations of two-digit numbers. Psychology Science, 47(1), 52-72.

Pinhas, M., Pothos, E. M., \& Tzelgov, J. (2013). Zooming in and out from the mental number line: Evidence for a number range effect. Journal of Experimental Psychology: Learning, Memory, and Cognition, 39(3), 972-976.

Poltrock, S. E., \& Schwartz, D. R. (1984). Comparative judgments of multi-digit numbers. Journal of Experimental Psychology: Learning, Memory, and Cognition, 10(1), 32-45.

Reynvoet, B., \& Brysbaert, M. (1999). Single-digit and two-digit Arabic numerals address the same semantic number line. Cognition, 72(2), 191-201.

Sasanguie, D., Defever, E., Van den Bussche, E., \& Reynvoet, B. (2011). The reliability of and the relation between non-symbolic numerical distance effects in comparison, same-different judgments and priming. Acta Psychologica, 136(1), 73-80.

Shaki, S., \& Fischer, M. H. (2013). Your neighbors define your value: A study of spatial bias in number comparison. Acta Psychologica, 142(3), 308-313.

Szücs, D., \& Soltész, F. (2010). Event-related brain potentials to violations of arithmetic syntax represented by place value structure. Biological Psychology, 84(2), 354-367.

Turconi, E., Campbell, J. I. D., \& Seron, X. (2006). Numerical order and quantity processing in number comparison. Cognition, 98(3), 273-285.

Tzelgov, J., Meyer, J., \& Henik, A. (1992). Automatic and intentional processing of 
numerical information. Journal of Experimental Psychology: Learning, Memory, and Cognition, 18(1), 166-179.

Varma, K., \& Karl, S. R. (2013). Understanding decimal proportions: Discrete representations, parallel access, and privileged processing of zero. Cognitive Psychology, 66(3), 283-301.

Verguts, T., \& De Moor, W. (2005). Two-digit comparison: Decomposed, holistic, or hybrid? Experimental Psychology, 52(3), 195-200.

Zhang, J., \& Wang, H. (1995). The integration of internal and external information in numerical tasks. Proceedings of the Seventeenth Annual Conference of the Cognitive Science Society (pp. 791-795). Hillsdale, NJ: Erlbaum.

Zhang, J., \& Wang, H. (2005). The effect of external representations on numeric tasks. The Quarterly Journal of Experimental Psychology Section A: Human Experimental Psychology, 58(5), 817-838.

Zhou, X. L., Chen, C. S., Chen, L., \& Dong, Q. (2008). Holistic or compositional representation of two-digit numbers? Evidence from the distance, magnitude, and SNARC effects in a number-matching task. Cognition, 106(3), 1525-1536. 


\section{Table 1}

Mean RTs and accuracy on different conditions for integers.

\begin{tabular}{cccccc}
\hline & \multicolumn{2}{c}{ Small decade distance } & \multicolumn{2}{c}{ Large decade distance } \\
\hline & Small unit & Large unit & Small unit & Large unit \\
& distance & distance & distance & distance \\
\hline RTs (ms) & Compatible & $604 \pm 75$ & $614 \pm 86$ & $549 \pm 63$ & $550 \pm 67$ \\
& Incompatible & $614 \pm 84$ & $624 \pm 93$ & $546 \pm 66$ & $555 \pm 64$ \\
ACC (\%) & Compatible & $95.15 \pm 3.47$ & $94.33 \pm 4.79$ & $99.52 \pm 1.16$ & $99.00 \pm 1.07$ \\
& Incompatible & $93.74 \pm 4.17$ & $93.78 \pm 4.01$ & $99.33 \pm 1.07$ & $98.15 \pm 2.09$ \\
\hline
\end{tabular}


Table 2

Mean RTs and accuracy on different conditions for pure decimals in Experiment 2.

\begin{tabular}{cccccc}
\hline & & \multicolumn{2}{c}{ Small tenths distance } & \multicolumn{2}{c}{ Large tenths distance } \\
\cline { 3 - 6 } \cline { 3 - 5 } & & distance & distance & distance & distance \\
\hline RT & Compatible & $648 \pm 65$ & $664 \pm 72$ & $590 \pm 63$ & $591 \pm 62$ \\
(ms) & Incompatible & $653 \pm 62$ & $662 \pm 70$ & $589 \pm 64$ & $594 \pm 59$ \\
ACC & Compatible & $93.48 \pm 5.34$ & $93.48 \pm 5.20$ & $99.14 \pm 1.71$ & $99.10 \pm 1.09$ \\
$(\%)$ & Incompatible & $92.90 \pm 4.40$ & $95.05 \pm 4.94$ & $99.48 \pm 0.98$ & $98.67 \pm 1.77$ \\
\hline
\end{tabular}


Table 3

Mean RTs and accuracy on different conditions for mixed decimals in Experiment 3.

\begin{tabular}{cccccc}
\hline & & \multicolumn{2}{c}{ Small unit distance } & \multicolumn{2}{c}{ Large unit distance } \\
\cline { 3 - 6 } & & Small tenths & Large tenths & Small tenths & Large tenths \\
\hline RT & Compatible & $599 \pm 90$ & $608 \pm 97$ & $541 \pm 75$ & $545 \pm 80$ \\
$(\mathrm{~ms})$ & Incompatible & $604 \pm 92$ & $604 \pm 92$ & $546 \pm 83$ & $548 \pm 82$ \\
\hline ACC & Compatible & $95.60 \pm 3.64$ & $95.93 \pm 3.88$ & $99.70 \pm 0.79$ & $99.57 \pm 0.90$ \\
$(\%)$ & Incompatible & $95.87 \pm 4.35$ & $95.77 \pm 3.74$ & $99.50 \pm 1.04$ & $98.97 \pm 1.40$ \\
\hline
\end{tabular}




\section{Table 4}

Mean RTs and accuracy on different conditions for pure decimals in Experiment 4.

\begin{tabular}{lccccc}
\hline & & \multicolumn{2}{c}{ Small tenths distance } & \multicolumn{2}{c}{ Large tenths distance } \\
\cline { 3 - 6 } & & Small & Large & Small & Large \\
\hline 20\% ratio & & & & \\
RT (ms) & Compatible & $697 \pm 88$ & $695 \pm 90$ & $639 \pm 71$ & $616 \pm 78$ \\
& Incompatible & $707 \pm 92$ & $721 \pm 100$ & $646 \pm 81$ & $638 \pm 81$ \\
ACC (\%) & Compatible & $94.47 \pm 5.87$ & $94.71 \pm 6.73$ & $99.63 \pm 1.10$ & $99.45 \pm 1.31$ \\
$70 \%$ ratio & & & & & \\
RT (ms) & Compatible & $729 \pm 55$ & $711 \pm 59$ & $663 \pm 53$ & $656 \pm 51$ \\
& Incompatible & $796 \pm 60$ & $781 \pm 47$ & $696 \pm 56$ & $709 \pm 54$ \\
ACC (\%) & Compatible & $95.65 \pm 3.76$ & $97.30 \pm 3.78$ & $99.80 \pm 0.89$ & $99.55 \pm 1.39$ \\
\hline
\end{tabular}


Table 5

Mean RTs and accuracy on different conditions for mixed decimals in Experiment 4.

\begin{tabular}{lccccc}
\hline \multicolumn{2}{c}{ Small unit distance } & \multicolumn{2}{c}{ Large unit distance } \\
& & Small tenths & Large tenths & Small tenths & Large tenths \\
& distance & distance & distance & distance \\
\hline 20\% ratio & & & & \\
RT & Compatible & $643 \pm 65$ & $640 \pm 73$ & $592 \pm 73$ & $570 \pm 61$ \\
(ms) & Incompatible & $665 \pm 85$ & $675 \pm 89$ & $602 \pm 77$ & $593 \pm 79$ \\
ACC & Compatible & $97.10 \pm 3.60$ & $96.08 \pm 4.78$ & $99.32 \pm 1.74$ & $99.65 \pm 1.08$ \\
(\%) & Incompatible & $94.82 \pm 4.88$ & $89.95 \pm 8.45$ & $99.10 \pm 1.62$ & $99.27 \pm 1.52$ \\
\hline $70 \%$ ratio & & & & $615 \pm 46$ \\
RT & Compatible & $681 \pm 60$ & $678 \pm 64$ & $627 \pm 45$ & $659 \pm 67$ \\
(ms) & Incompatible & $741 \pm 77$ & $739 \pm 78$ & $656 \pm 55$ & $100.00 \pm 0.00$ \\
ACC & Compatible & $94.80 \pm 4.21$ & $97.12 \pm 3.15$ & $99.80 \pm 0.89$ & $95.42 \pm 4.44$ \\
\hline (\%) & Incompatible & $85.87 \pm 8.24$ & $85.55 \pm 8.91$ & $97.02 \pm 3.21$ & \\
\hline
\end{tabular}

